\title{
ANNOUNCEMENT
}

\section{International Commission on Trichinellosis}

The 6th International Conference on Trichinellosis will take place between 8 th and 12th July 1984 at the Far Hills Inn in Val Morine, Québec, Canada. PLEASE NOTE THE CHANGE IN DATES FROM THOSE PREVIOUSLY ANNOUNCED

Further information from:

Dr. Charles E. Tanner, Institute of Parasitology,

Box 231, Macdonald College of McGill University, 21, 111 Lakeshore Road, Ste. Anne-de-Bellevue, Québec, Canada H9X 1 CO. 